\section{Isolated Renal Arteritis With Infarction Identified After SARS-CoV-2 Vaccine}

\author{
Akiko Masumoto, MD; \\ Hiroyuki Yamamoto, MD, PhD; \\ Yasuyo Taniguchi, $\mathrm{MD}, \mathrm{PhD}$; \\ Hiroya Kawai, MD, PhD; \\ Tomofumi Takaya, MD, PhD
}

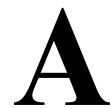

48-year-old healthy man, who had received the first- and second-dose SARS-CoV-2 vaccine (BNT162b2 mRNA: Pfizer-BioNTech) 25 days and 4 days prior, presented with persistent high-grade fever and sudden-onset left-sided back pain for 3 days. Contrast-enhanced computed tomography revealed left renal infarction with thrombus in the mid-left renal artery (Figure A-D). Blood test, transesophageal echocardiography and gadolinium-enhanced cardiac magnetic resonance imaging (MRI) found no abnormalities, including coagulopathy, collagen or malignant disease, and cardiogenic thromboembolism. A 2-week ECG monitoring detected no arrhythmia. Although no signs of atherosclerotic degeneration or medium-vessel vasculitis were detected, abdominal MRI suggested a localized left renal arteritis (Figure E,F). Finally, the findings suggested that renal infarction could have been caused by thrombus formation at the site of localized renal arteritis. Intravenous heparin and acetaminophen were effective in preventing further fever and thrombotic events, and he was discharged without renal dysfunction (Figure G). Renal ultrasound before discharge confirmed no residual stenosis but decreased left renal blood flow remained (Figure $\mathbf{H}$ ).

A recent case series identified increased risk of arterial thromboembolism among 30 million people who underwent SARS-CoV-2 vaccination. ${ }^{1}$ Increased risks of immune- mediated disease and myocarditis after vaccination have been reported. ${ }^{2,3}$ Our case suggest that isolated renal arteritis with infarction could be potentially associated with SARS-CoV-2 vaccine-related immune-mediated disease. For the possibility of unknown SARS-CoV-2 vaccinerelated adverse events, multimodal imaging can be useful to guide diagnosis and treatment. Although the benefits of SARS-CoV-2 vaccination are unarguably clear, its safety monitoring should be continued while accumulating rare vaccine-related adverse events.

\section{Conflict of Interest / Acknowledgments}

None.

\section{References}

1. Hippisley-Cox J, Patone M, Mei XW, Saatci D, Dixon S, Khunti $\mathrm{K}$, et al. Risk of thrombocytopenia and thromboembolism after covid-19 vaccination and SARS-CoV-2 positive testing: Selfcontrolled case series study. BMJ 2021; 374: n1931.

2. Watad A, De Marco G, Mahajna H, Druyan A, Eltity M, Hijazi $\mathrm{N}$, et al. Immune-mediated disease flares or new-onset disease in 27 subjects following mRNA/DNA SARS-CoV-2 vaccination. Vaccines (Basel) 2021; 9: 435.

3. Sakaguchi S, Fujimoto N, Ichikawa K, Izumi D, Katsuta K, Takafuji M, et al. Myopericarditis after COVID-19 mRNA vaccination. Circ J, doi:10.1253/circj.CJ-21-0683.

Received December 20, 2021; revised manuscript received December 29, 2021; accepted January 10, 2022; J-STAGE Advance Publication released online January 29, 2022 Time for primary review: 9 days

Division of Cardiovascular Medicine, Hyogo Brain and Heart Center, Himeji (A.M., H.Y., Y.T., H.K., T.T.); Department of Exploratory and Advanced Search in Cardiology, Kobe University Graduate School of Medicine, Kobe (H.K., T.T.), Japan

Mailing address: Hiroyuki Yamamoto, MD, PhD, Division of Cardiovascular Medicine, Hyogo Brain and Heart Center, SaishoKou-520, Himeji 670-0981, Japan. E-mail: y0493589m@hotmail. co.jp

All rights are reserved to the Japanese Circulation Society. For permissions, please e-mail: cj@j-circ.or.jp ISSN-1346-9843

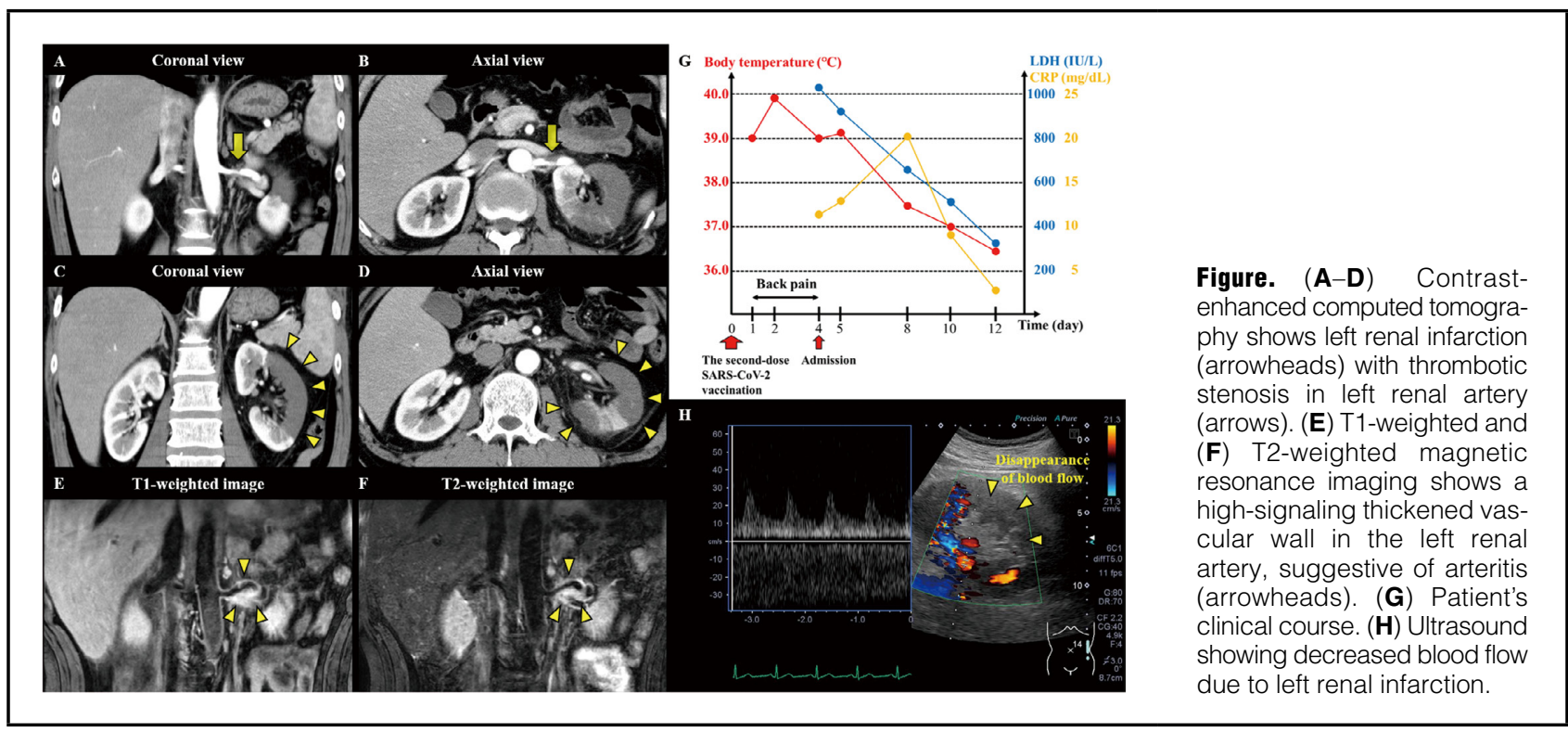

